\title{
WEAKLY NONLINEAR BOUNDARY VALUE PROBLEM FOR A MATRIX DIFFERENTIAL EQUATION
}

\author{
S. CHUIKO
}

Received 06 September, 2014

\begin{abstract}
We set forth solvability conditions and construction of the generalized Green operator for Noetherian linear boundary value problem for the matrix differential equations and solvability conditions and the constructive scheme for constructing solutions of nonlinear Noetherian boundary value problem for matrix differential equation. We show that the principal results in the theory of weakly nonlinear periodic oscillations remain valid for nonlinear Noetherian boundary value problem for matrix differential equation. The study is illustrated by the periodic problem for a Duffing-type matrix differential equation.
\end{abstract}

2010 Mathematics Subject Classification: 34B10; 34B15

Keywords: boundary value problems, matrix differential equations

\section{INTRODUCTION}

We investigate the problem of the determination of conditions for the existence of solution [5]

$$
Z(t, \varepsilon): Z(\cdot, \varepsilon) \in \mathbb{C}^{1}[a ; b], \quad Z(t, \cdot) \in \mathbb{C}\left[0 ; \varepsilon_{0}\right], \quad Z(t, \varepsilon) \in \mathbb{R}^{\alpha \times \beta}
$$

of the matrix differential system

$$
Z^{\prime}(t, \varepsilon)=A Z(t, \varepsilon)+Z(t, \varepsilon) B+F(t)+\varepsilon \Phi(Z(t, \varepsilon), t, \varepsilon),
$$

that satisfy the boundary condition $[3,4,9]$

$$
\mathscr{L} Z(\cdot, \varepsilon)=\mathcal{A}, \quad \mathcal{A} \in \mathbb{R}^{\delta \times \gamma}
$$

and the construction of this solution. We seek a solution of the Noetherian $(\alpha \neq \beta \neq$ $\delta \neq \gamma$ ) matrix boundary value problems (1.1), (1.2) in a small neighbourhood of the generating problem

$$
Z_{0}^{\prime}(t)=A Z_{0}(t)+Z_{0}(t) B+F(t), \quad \mathscr{L} Z_{0}(\cdot)=\mathcal{A},
$$

The author was supported in part by the State Fund for Fundamental Research of Ukraine, Grant No. 0109 U000381. 
where $A \in \mathbb{R}^{\alpha \times \alpha}$ and $B \in \mathbb{R}^{\beta \times \beta}$ are real constant matrices, $F(t) \in \mathbb{C}[a, b]$ is an $\alpha \times \beta$ matrix whose elements are real functions continuous on the segment $[a, b], \mathscr{L} Z(\cdot, \varepsilon)$ is a linear bounded matrix functional:

$$
\mathscr{L} Z(\cdot, \varepsilon): \mathbb{C}^{1}[a ; b] \rightarrow \mathbb{R}^{\delta \times \gamma} .
$$

Assume that the nonlinear operator

$$
\Phi(Z(t, \varepsilon), t, \varepsilon): \mathbb{R}^{\alpha \times \beta} \rightarrow \mathbb{R}^{\alpha \times \beta}
$$

is continuously differentiable with respect to unknown $Z(t, \varepsilon)$ in a small neighbourhood of the generating solutions and with respect to the small parameter $\varepsilon$ in a small positive neighbourhood of zero. In addition, we assume that the matrix function $\Phi(Z(t, \varepsilon), t, \varepsilon)$ is continuous in the independent variable $t$ on the segment $[a, b]$.

The structure of the set of solution of the generating matrix boundary value problem (1.3) was studied by Bellman [1]. In [4] the authors established solvability conditions and construction of the generalized Green operator in case $\alpha=\beta$ for the periodical matrix boundary value problem (1.3). Denote by $U(t)$ and $V(t)-$ normal fundamental matrices:

$$
U^{\prime}(t)=A U(t), \quad U(a)=I_{\alpha}, \quad V^{\prime}(t)=B V(t), \quad V(a)=I_{\beta} .
$$

The matrix Cauchy problem [1]

$$
Z^{\prime}(t)=A Z(t)+Z(t) B, \quad Z(a)=\Theta
$$

has a solution

$$
W(t, \Theta)=U(t) \cdot \Theta \cdot V(t), \quad \Theta \in \mathbb{R}^{\alpha \times \beta} .
$$

The general solution

$$
Z(t, \Theta)=W(t, \Theta)+K[F(s)](t), \quad \Theta \in \mathbb{R}^{\alpha \times \beta}
$$

of the matrix Cauchy problem [4]

$$
Z^{\prime}(t)=A Z(t)+Z(t) B+F(t), \quad Z(a)=\Theta
$$

is determined by Green operator of the matrix Cauchy problem

$$
K[F(s)](t):=\int_{a}^{t} U(t) U^{-1}(s) F(s) V(t) V^{-1}(s) d s .
$$

2. SOLVABILITY CONDITIONS FOR WEAKLY NONLINEAR

BOUNDARY VALUE PROBLEM FOR MATRIX DIFFERENTIAL EQUATION

Substituting general solution of the Cauchy problem $Z(a)=\Theta$ for the generating matrix differential system (1.3)

$$
Z_{0}(t, \Theta)=W(t, \Theta)+K[F(s)](t), \quad \Theta \in \mathbb{R}^{\alpha \times \beta}
$$


in the boundary condition (1.3), we get matrix equation

$$
\mathscr{L} Z_{0}(\cdot, \Theta)=\mathcal{A}-\mathscr{L} K[F(s)](\cdot) .
$$

Let $\Xi^{(j)} \in \mathbb{R}^{\alpha \times \beta}, j=1,2, \ldots \alpha \cdot \beta$ be standard basis of the space $\mathbb{R}^{\alpha \times \beta}$ and let

$$
\Theta=\sum_{j=1}^{\alpha \cdot \beta} \Xi^{(j)} c_{j}, \quad c_{j} \in \mathbb{R}^{1}, \quad j=1,2, \ldots, \alpha \cdot \beta .
$$

Then

$$
\mathscr{L} W(\cdot, \Theta)=\sum_{j=1}^{\alpha \cdot \beta} \mathscr{L} U(\cdot) \Xi^{(j)} V(\cdot) c_{j} .
$$

We introduce the linear operator [9] $\mathcal{M}[\mathscr{B}]: \mathbb{R}^{m \times n} \rightarrow \mathbb{R}^{m \cdot n}$, which assigns a matrix $\mathscr{B} \in \mathbb{R}^{m \times n}$ column vector $\mathcal{M}[\mathscr{B}] \in \mathbb{R}^{m \cdot n}$, consisting of the columns of this matrix. Further, we introduce the inverse operator [9]

$$
\mathcal{M}^{-1}\{\mathcal{M}[\mathscr{B}]\}: \mathbb{R}^{m \cdot n} \rightarrow \mathbb{R}^{m \times n},
$$

which assigns a column vector $\mathcal{M}[\mathcal{B}] \in \mathbb{R}^{m \cdot n}$ matrix $\mathscr{B} \in \mathbb{R}^{m \times n}$. Applying the operator $\mathcal{M}$ to the matrix equation (2.1), we obtain the following system equations

$$
\mathcal{Q} c=\mathcal{M}[\mathcal{A}]-\mathcal{M}\{\mathscr{L} K[F(s)](\cdot)\}
$$

where

$$
\mathcal{Q}:=\left[\mathcal{M}\left[\mathcal{Q}^{(1)}\right] \mathcal{M}\left[\mathcal{Q}^{(2)}\right] \ldots \mathcal{M}\left[\mathcal{Q}^{(\alpha \cdot \beta)}\right]\right] \in \mathbb{R}^{\delta \cdot \gamma \times \alpha \cdot \beta}
$$

and

$$
\mathcal{Q}^{(j)}:=\mathscr{L} U(\cdot) \Xi^{(j)} V(\cdot) \in \mathbb{R}^{\beta \times \delta}, \quad j=1,2, \ldots, \alpha \cdot \beta .
$$

Under the condition $[5,9,10]$

$$
P_{\mathcal{Q}_{d}^{*} \mathcal{M}}\{\mathcal{A}-\mathscr{L} K[F(s)](\cdot)\}=0,
$$

the generating problem (1.3) has $r$ linearly independent solution

$$
Z_{0}\left(t, \Theta_{r}\right)=W\left(t, \Theta_{r}\right)+G[F(s) ; \mathcal{A}](t), \quad \Theta_{r}:=\mathcal{M}^{-1}\left[P_{Q_{r}} c_{r}\right] .
$$

Here $W(t, \Theta)$ is the normal $(W(a, \Theta)=\Theta)$ fundamental matrix of the homogeneous part of the generating matrix differential system (1.3), $W\left(t, \Theta_{r}\right)$ is an $\alpha \times \beta$ matrix composed of $r$ linearly independent solution of the homogeneous part of the generating matrix boundary value problem (1.3), $P_{\mathcal{Q}_{r}}$ is $\alpha \cdot \beta \times r$ matrix composed of $r$ linearly independent columns of the $\alpha \cdot \beta \times \alpha \cdot \beta$ matrix orthoprojector 
$P_{\mathcal{Q}}: \mathbb{R}^{\alpha \cdot \beta \times \alpha \cdot \beta} \rightarrow \mathbb{N}(\mathcal{Q}) ; P_{Q_{d}^{*}}$ is $d \times \delta \cdot \gamma$ matrix composed of $d$ linearly independent rows of the $\delta \cdot \gamma \times \delta \cdot \gamma$ matrix orthoprojector $P_{Q^{*}}: \mathbb{R}^{\delta \cdot \gamma} \rightarrow \mathbb{N}\left(Q^{*}\right), Q^{+}$is the pseudoinverse Moore-Penrose matrix [5, 13],

$$
G[F(s) ; \mathcal{A}](t):=W\left\{t, \mathcal{M}^{-1}\left\{\mathcal{Q}^{+} M[\mathcal{A}-\mathscr{L} K[F(s)](\cdot)]\right\}\right\}+K[F(s)](t)
$$

is the generalized Green operator [10] for generating problem (1.3),

$$
W\left(t, \Theta_{r}\right):=\sum_{k=1}^{r} U(t) \cdot \Xi^{\left(j_{k}\right)} V(t) \cdot c_{j_{k}}, \quad \Theta_{r}=\sum_{k=1}^{r} \Xi^{\left(j_{k}\right)} c_{j_{k}}
$$

— the general solution of the homogeneous part of the matrix boundary-value problem (1.3).

2.1. Generalized Green operator for Noetherian linear boundary value problem for the matrix differential equations

The following lemma gives necessary and sufficient conditions for the solvability and construction of the generalized Green operator for Noetherian linear boundary value problem for the matrix differential equations (1.3).

Lemma 1. The inhomogeneous boundary value problem for the matrix differential equations (1.3) is solvable if and only if

$$
P_{Q_{d}^{*} \mathcal{M}}\{\mathcal{A}-\mathscr{L} K[F(s)](\cdot)\}=0 .
$$

In this case, boundary value problem for the matrix differential equations (1.3) possesses $r$ linearly independent solution

$$
Z_{0}\left(t, \Theta_{r}\right)=W\left(t, \Theta_{r}\right)+G[F(s) ; \mathcal{A}](t), \quad \Theta_{r}=\sum_{k=1}^{r} \Xi^{\left(j_{k}\right)} c_{j_{k}} .
$$

The proved Lemma 1 generalizes analogous statements for various boundary value problem for the differential equations [5]. The proved Lemma 1 also generalizes analogous statements for periodic boundary value problem for a matrix Riccati equation [4]. Provided $P_{Q^{*}} \neq 0$, we say that the boundary value problem (1.3) there is a critical case, and the problem (1.3) solvable only for those inhomogeneities of $F(t)$ and $\mathcal{A}$, for which the condition (2.3). Provided $P_{Q^{*}}=0$ for the boundary value problem (1.3) holds non-critical case, and the problem (1.3) is solvable for any inhomogeneities of $F(t)$ and $\mathcal{A}$.

The nonperturbed problem (1.3) has the solution $Z_{0}\left(t, \Theta_{r}\right)$. We seek a solution $Z(t, \varepsilon)$ of problem $(1.1),(1.2)$ such that $Z(\cdot, \varepsilon) \in \mathbb{C}^{1}[a ; b], Z(t, \cdot) \in \mathbb{C}\left[0 ; \varepsilon_{0}\right]$ and $Z(t, 0)=Z_{0}\left(t, \Theta_{r}\right)$. In problem (1.3), (1.2) we perform the substitution $Z(t, \varepsilon)=$ $Z_{0}\left(t, \Theta_{r}\right)+X(t, \varepsilon)$. For

$$
X(\cdot, \varepsilon) \in \mathbb{C}^{1}[a ; b], \quad X(t, \cdot) \in \mathbb{C}\left[0 ; \varepsilon_{0}\right], \quad X(t, \varepsilon) \in \mathbb{R}^{\alpha \times \beta},
$$


we obtain the problem

$$
X^{\prime}(t, \varepsilon)=A X(t, \varepsilon)+X(t, \varepsilon) B+\varepsilon \Phi(Z(t, \varepsilon), t, \varepsilon), \quad \mathscr{L} X(\cdot, \varepsilon)=0 .
$$

Considering the nonlinearity in problem (2.4) as nonhomogeneous term, applying Lemma 1 to problem (2.4), and taking account relation (2.3), we find that on the set of functions $X(t, \varepsilon)$ such that $X(t, 0) \equiv 0$, boundary value problem (2.4) is equivalent to the operator equation

$$
X(t, \varepsilon)=\varepsilon G\left[\Phi\left(Z_{0}\left(s, \Theta_{r}\right)+X(s, \varepsilon), s, \varepsilon\right) ; 0\right](t),
$$

which is the fixed point for the operator

$$
\mathcal{E}: \mathcal{E}[X]:=\varepsilon G\left[\Phi\left(Z_{0}\left(s, \Theta_{r}\right)+X(s, \varepsilon), s, \varepsilon\right) ; 0\right](t)
$$

defined on the closed set

$$
\begin{aligned}
\Omega=\left\{X(t, \varepsilon): X(\cdot, \varepsilon) \in \mathbb{C}^{1}[a ; b], \quad X(t, \cdot) \in \mathbb{C}\left[0 ; \varepsilon_{0}\right],\right. \\
X(t, 0) \equiv 0, \quad\|X(t, \varepsilon)\| \leq q=\mathrm{const}\}
\end{aligned}
$$

in the complete space of continuously differentiable matrix functions $X(t, \varepsilon)$ on $[a ; b]$. We can readily see that there exists an $\varepsilon_{*}, 0<\varepsilon_{*}<\varepsilon_{0}$, such that the operator $\mathscr{E}$ satisfies all assumptions of the Banach theorem on the set $\Omega$ for all $0 \leq \varepsilon<\varepsilon_{*}$. By that theorem, the operator equation (2.5) has at least one solution $X(t, \varepsilon) \in \Omega$. This solution can be found with the use of the iterative process

$$
X_{k+1}(t, \varepsilon)=\varepsilon G\left[\Phi\left(Z_{0}\left(s, \Theta_{r}\right)+X_{k}(s, \varepsilon), s, \varepsilon\right) ; 0\right](t), \quad k=0,1,2, \ldots
$$

convergent on $\left[0 ; \varepsilon_{*}\right]$. We have thereby justified the following assertion.

Theorem 1. In the noncritical case, boundary value problem (1.1), (1.2) has a solution

$$
Z(t, \varepsilon)=Z_{0}\left(t, \Theta_{r}\right)+X(t, \varepsilon), \quad X(\cdot, \varepsilon) \in \mathbb{C}^{1}[a ; b], \quad X(t, \cdot) \in \mathbb{C}\left[0 ; \varepsilon_{0}\right]
$$

such that $X(t, 0) \equiv 0$. This solution can be found with the use of the iterative process

$$
Z_{k+1}(t, \varepsilon)=Z_{0}\left(t, \Theta_{r}\right)+X_{k+1}(t, \varepsilon), \quad k=0,1,2, \ldots,
$$

where $X_{k}(t, \varepsilon)$ are given by (2.6).

The proved Theorem 1 generalizes analogous statements for various boundary value problem for the differential equations [5]. Let us now analyze the existence of solution boundary value problem (1.1), (1.2) such that, for $\varepsilon=0$, these solutions become one of the solutions $Z_{0}\left(t, \Theta_{r}\right)$ of the unperturbed problem (1.3) in the critical case. 
Theorem 2. (A necessary condition). Let the inhomogeneities $F(t)$ and $\mathcal{A}$ satisfy criterion (2.3) for the solvability of the nonperturbed problem (1.3), and let boundary value problem (1.1), (1.2) have a solution $Z(t, \varepsilon)$ such that

$$
Z(\cdot, \varepsilon) \in \mathbb{C}^{1}[a ; b], \quad Z(t, \cdot) \in \mathbb{C}\left[0 ; \varepsilon_{0}\right]
$$

and $Z(t, 0)=Z_{0}\left(t, \Theta_{r}\right)$. Then the matrix $\Theta_{r} \in \mathbb{R}^{\alpha \times \beta}$ satisfies the equation

$$
P_{Q_{d}^{*} \mathcal{M}}\left\{\mathscr{L} K\left[\Phi\left(Z_{0}\left(s, \Theta_{r}\right), s, 0\right)\right](\cdot)\right\}=0 .
$$

Proof. Applying solvability criterion (2.3) to the function

$$
F(t)+\varepsilon \Phi(Z(t, \varepsilon), t, \varepsilon)
$$

occurring in (1.1), we obtain

$$
P_{Q_{d}^{*} \mathcal{M}}\{\mathscr{L} K[F(s)+\varepsilon \Phi(Z(s, \varepsilon), s, \varepsilon)](\cdot)\}=0 .
$$

Since $\mathcal{M}$ and $\mathscr{L}$ are a linear operators and the inhomogeneities $F(t)$ and $\mathcal{A}$ satisfy criterion (2.3) for the solvability of the unperturbed problem (1.3), we have

$$
P_{\mathcal{Q}_{d}^{*}} \mathcal{M}\left\{\mathscr{L} K\left[\Phi\left(Z_{0}\left(s, \Theta_{r}\right)+X(s, \varepsilon), s, \varepsilon\right)\right](\cdot)\right\}=0 .
$$

By setting $\varepsilon=0$ in the last relation, we obtain (2.7). Following [4,5,14,17], we refer to equation (2.7) as an equation for generating constants.

The proved Theorem 2 generalizes analogous statements for various boundary value problem for the differential equations $[5,14,17]$.

To derive a sufficient solvability conditions for boundary value problem (1.1), (1.2), we perform the substitution $Z(t, \varepsilon)=Z_{0}\left(t, \Theta_{r}^{*}\right)+X(t, \varepsilon)$, where the matrix $\Theta_{r}^{*} \in \mathbb{R}^{\alpha \times \beta}$ satisfies the necessary and sufficient solvability condition (2.8). Using the continuous differentiability of the function $\Phi[Z(t, \varepsilon), t, \varepsilon]$ with respect to unknown $Z(t, \varepsilon)$, in a small neighbourhood of the generating solutions and with respect to the small parameter $\varepsilon$ in a small positive neighbourhood of zero, we expand this function in the neighbourhood of the points $X(t, \varepsilon)=0$ and $\varepsilon=0$ as follows [15]:

$$
\begin{aligned}
\Phi[Z(t, \varepsilon), t, \varepsilon]=\Phi\left[Z_{0}\left(t, \Theta_{r}^{*}\right), t, 0\right]+D\left[Z_{0}\left(t, \Theta_{r}^{*}\right) ; X(t, \varepsilon)\right] \\
+R\left[Z_{0}\left(t, \Theta_{r}^{*}\right)+X(t, \varepsilon), t, \varepsilon\right],
\end{aligned}
$$

where the differential $D\left[Z_{0}\left(t, \Theta_{r}^{*}\right) ; X(t, \varepsilon)\right]$ of the function $\Phi[Z(t, \varepsilon), t, \varepsilon]$ is a linear operator

$$
D\left[Z_{0}\left(t, \Theta_{r}^{*}\right) ; \lambda X^{(a)}(t, \varepsilon)+\mu X^{(b)}(t, \varepsilon)\right]
$$




$$
=\lambda D\left[Z_{0}\left(t, \Theta_{r}^{*}\right) ; X^{(a)}(t, \varepsilon)\right]+\mu D\left[Z_{0}\left(t, \Theta_{r}^{*}\right) ; X^{(b)}(t, \varepsilon)\right], \quad \lambda, \mu \in \mathbb{R}^{1}
$$

with respect to unknown $X(t, \varepsilon)$. Since the remainder $R\left(Z_{0}\left(t, \Theta_{r}^{*}\right)+X(t, \varepsilon), t, \varepsilon\right)$ of the expansion of the function $\Phi[Z(t, \varepsilon), t, \varepsilon]$ has a higher order of smallness with respect to the unknown $X(t, \varepsilon)$ in the neighbourhood of the points $X(t, \varepsilon)=0$ and $\varepsilon=0$ than the first two terms of expansion, we have

$$
R\left[Z_{0}\left(t, \Theta_{r}^{*}\right)+X(t, \varepsilon), t, 0\right] \equiv 0 .
$$

For the function $X=X(t, \varepsilon)$ we obtain the problem

$$
\begin{gathered}
X^{\prime}=A X+X B+\varepsilon \Phi\left(Z_{0}, t, 0\right)+\varepsilon D\left[Z_{0} ; X\right]+\varepsilon R\left(Z_{0}+X, t, \varepsilon\right), \\
\mathscr{L} X(\cdot, \varepsilon)=0,
\end{gathered}
$$

where

$D\left[Z_{0} ; X\right]=D\left[Z_{0}\left(t, \Theta_{r}^{*}\right) ; X(t, \varepsilon)\right], R\left(Z_{0}+X, t, \varepsilon\right)=R\left(Z_{0}\left(t, \Theta_{r}^{*}\right)+X(t, \varepsilon), t, \varepsilon\right)$.

We intend to construction an operator system equivalent to problem (2.9), (2.10) on the set of matrix functions $X=X(t, \varepsilon)$ such that $X(\cdot, \varepsilon) \in \mathbb{C}^{1}[a ; b], X(t, \cdot) \in \mathbb{C}\left[0 ; \varepsilon_{0}\right]$, and $X(t, 0)=0$. Applying Lemma 1 to the boundary value problem (2.9), (2.10), we obtain

$$
\begin{aligned}
X(t, \varepsilon)= & W\left(t, \Theta_{r}(\varepsilon)\right)+X^{(1)}(t, \varepsilon), \Theta_{r}(\varepsilon)=\mathcal{M}^{-1}\left[c_{r}(\varepsilon)\right], \\
X^{(1)}(t, \varepsilon)= & \varepsilon G\left[\Phi\left(Z_{0}\left(s, \Theta_{r}^{*}\right)+X(s, \varepsilon), s, \varepsilon\right) ; 0\right](t), \\
\mathcal{B}_{0} \cdot c_{r}(\varepsilon)= & -P_{Q_{d}^{*} \mathcal{M}}\left\{\mathscr{L} K\left\{D\left[Z_{0}\left(s, \Theta_{r}^{*}\right) ; X^{(1)}(s, \varepsilon)\right]\right\}(\cdot)\right. \\
& \left.+\mathscr{L} K\left[R\left(Z_{0}\left(s, \Theta_{r}^{*}\right)+X(s, \varepsilon), s, \varepsilon\right)\right](\cdot)\right\},
\end{aligned}
$$

where

and

$$
\mathscr{B}_{0}:=\left[\begin{array}{lllll}
\mathscr{B}_{0}^{(1)} & \ldots & \mathcal{B}_{0}^{(j)} & \ldots & \mathcal{B}_{0}^{(r)}
\end{array}\right] \in \mathbb{R}^{d \times r}, \quad j=1,2, \ldots r
$$

$$
\mathscr{B}_{0}^{(j)}:=P_{Q_{d}^{*}} \mathcal{M}\left\{\mathscr{L} K\left\{D\left[Z_{0}\left(s, \Theta_{r}\right) ; U(s) \cdot \Xi^{(j)} \cdot V(s)\right]\right\}(\cdot) \in \mathbb{R}^{d} .\right.
$$

Equation (2.11) is solvable if an only if

$$
\begin{aligned}
P_{\mathscr{B}_{0}^{*}} P_{Q_{d}^{*} \mathcal{M}}\left\{\mathscr { L } K \left\{D \left[Z_{0}\left(s, \Theta_{r}^{*}\right)\right.\right.\right. & \left.\left.X^{(1)}(s, \varepsilon)\right]\right\}(\cdot) \\
& \left.+\mathscr{L} K\left[R\left(Z_{0}\left(s, \Theta_{r}^{*}\right)+X(s, \varepsilon), s, \varepsilon\right)\right](\cdot)\right\}=0 .
\end{aligned}
$$


For $P_{\mathcal{B}_{0}^{*}} P_{\mathcal{Q}_{d}^{*}}=0$ a solution of the boundary value problem (2.9), (2.10) is determined by the operator system

$$
\begin{aligned}
X(t, \varepsilon)= & W\left(t, \Theta_{r}(\varepsilon)\right)+X^{(1)}(t, \varepsilon), \quad \Theta_{r}(\varepsilon)=\mathcal{M}^{-1}\left[c_{r}(\varepsilon)\right], \\
X^{(1)}(t, \varepsilon)= & \varepsilon G\left[\Phi\left(Z_{0}\left(s, \Theta_{r}^{*}\right)+X(s, \varepsilon), s, \varepsilon\right) ; 0\right](t), \\
c_{r}(\varepsilon)= & -\mathcal{B}_{0}^{+} P_{\mathcal{Q}_{d}^{*}} \mathcal{M}\left\{\mathscr{L} K\left\{D\left[Z_{0}\left(s, \Theta_{r}^{*}\right) ; X^{(1)}(s, \varepsilon)\right]\right\}(\cdot)\right. \\
& \left.+\mathscr{L} K\left[R\left(Z_{0}\left(s, \Theta_{r}^{*}\right)+X(s, \varepsilon), s, \varepsilon\right)\right](\cdot)\right\},
\end{aligned}
$$

which is equivalent to the problem of the construction of a solution of the system of equation (2.9) with boundary condition (2.10). The operator system (2.12) is equivalent to the problem of the construction of a solution of equation

$$
X(t, \varepsilon)=\Psi\left[Z_{0}\left(s, \Theta_{r}^{*}\right)+X(s, \varepsilon)\right](t)
$$

on the set of functions $X(t, \varepsilon)$ that are equal to zero for $\varepsilon=0$; here

$$
\begin{aligned}
\Psi\left[Z_{0}\left(s, \Theta_{r}^{*}\right)+X(s, \varepsilon)\right](t) & :=W\left(t, \Theta_{r}(\varepsilon)\right)+X^{(1)}(t, \varepsilon) \\
& =W\left\{t, \mathcal{M}^{-1}\left[c_{r}(\varepsilon)\right]\right\}+\varepsilon G\left[\Phi\left(Z_{0}\left(s, \Theta_{r}^{*}\right)+X(s, \varepsilon), s, \varepsilon\right) ; 0\right](t) .
\end{aligned}
$$

The operator $\Psi[Z(s, \varepsilon)](t)$ is a superposition of an operator linear with respect to matrix function $\Phi(Z(t, \varepsilon), t, \varepsilon)$ that acts on the function $\Phi(Z(t, \varepsilon), t, \varepsilon)$ continuously differentiable with respect to $X(t, \varepsilon)$. Thus, $\Psi[Z(s, \varepsilon)](t)$ is a continuous bounded operator that acts from the space of real matrix functions $X(t, \varepsilon)$ continuous on the segment $[a, b]$ and $\left[0 ; \varepsilon_{0}\right]$ into itself. For $P_{\mathcal{B}_{0}^{*}} P_{Q_{d}^{*}}=0$ at least one solution of the boundary value problem (2.9), (2.10) can be determined by using an iteration process [15, p. 213]

$$
X_{k+1}(t, \varepsilon)=\Psi\left[Z_{0}\left(s, \Theta_{r}^{*}\right)+X_{k}(s, \varepsilon)\right](t), \quad \varepsilon \in\left[0, \varepsilon^{*}\right], \quad k=0,1,2 \ldots
$$

convergent on $\left[0 ; \varepsilon_{*}\right]$. We have thereby justified the following assertion.

Theorem 3. Suppose that the boundary value problem (1.3) corresponds to the critical case $P_{Q^{*}} \neq 0$ and let the inhomogeneities $F(t)$ and $\mathcal{A}$ satisfy criterion (2.3) for the solvability of the nonperturbed problem (1.3). Then, under condition $P_{\mathcal{B}_{0}^{*}} P_{Q_{d}^{*}}=0$ for every root $\Theta_{r}^{*} \in \mathbb{R}^{\alpha \times \beta}$ of equation for generating constants (2.7) boundary value problem (2.9), (2.10) has at least one solution

$$
X(\cdot, \varepsilon) \in \mathbb{C}^{1}[a ; b], \quad X(t, \cdot) \in \mathbb{C}\left[0 ; \varepsilon_{0}\right]
$$


that turns into the zero solution $X(t, 0)=0$ for $\varepsilon$. Moreover, boundary value problem (1.1), (1.2) has at least one solution

$$
Z(\cdot, \varepsilon) \in \mathbb{C}^{1}[a ; b], \quad Z(t, \cdot) \in \mathbb{C}\left[0 ; \varepsilon_{0}\right]
$$

that for $\left[0 ; \varepsilon_{*}\right]$ turns into the generating solution $Z(t, 0)=Z_{0}\left(t, \Theta_{r}^{*}\right)$. This solution can be determined by using an iteration process

$$
\begin{aligned}
Z_{k+1}(t, \varepsilon)= & Z_{0}\left(t, \Theta_{r}^{*}\right)+X_{k+1}(t, \varepsilon), \quad X_{k+1}(t, \varepsilon)=W\left(t, \Theta_{r}(\varepsilon)\right)+X_{k+1}^{(1)}(t, \varepsilon), \\
X_{k+1}^{(1)}(t, \varepsilon)= & \varepsilon G\left[\Phi\left(Z_{k}(s, \varepsilon), s, \varepsilon\right) ; 0\right](t), \quad \Theta_{r_{k+1}}(\varepsilon)=\mathcal{M}^{-1}\left[c_{r_{k+1}}(\varepsilon)\right], \\
c_{r_{k+1}}(\varepsilon)= & -\mathcal{B}_{0}^{+} P_{\mathcal{Q}_{d}^{*} \mathcal{M}}\left\{\mathscr{L} K\left\{D\left[Z_{0}\left(s, \Theta_{r}^{*}\right) ; X_{k}^{(1)}(s, \varepsilon)\right]\right\}(\cdot)\right. \\
& \left.+\mathscr{L} K\left[R\left(Z_{0}\left(s, \Theta_{r}^{*}\right)+X_{k}(s, \varepsilon), s, \varepsilon\right)\right](\cdot)\right\}, k=0,1,2 \ldots,
\end{aligned}
$$

which converges for $\left[0 ; \varepsilon_{*}\right]$.

The proved Theorem 3 generalizes analogous statements for various boundary value problem for the differential equations $[5,14,17]$. The proved Theorem 3 also generalizes analogous statements for periodic boundary value problem for a matrix Riccati equation [4]. The length $\varepsilon_{*}$ of the segment $\left[0, \varepsilon^{*}\right]$ on which the iterative procedure (2.14) converges to the required solution of the boundary value problem (1.1), (1.2) can be estimated by analogy with [7,8,14].

\section{MATRIX EQUATIONS OF DUFFING TYPE}

The requirements of Theorem 3 are satisfied in the problem of the construction of the $2 \pi-$ periodic solutions of matrix equations of Duffing type

$$
Z^{\prime}(t, \varepsilon)=A Z(t, \varepsilon)+Z(t, \varepsilon) B+F(t)+\varepsilon Z(t, \varepsilon) Z(t, \varepsilon)^{*} Z(t, \varepsilon),
$$

where

$$
A=\left(\begin{array}{ll}
1 & -2 \\
1 & -1
\end{array}\right), \quad B=\left(\begin{array}{cc}
-2 & -2 \\
4 & 2
\end{array}\right), \quad F(t)=\left(\begin{array}{cc}
\sin 5 t & 0 \\
0 & \cos 5 t
\end{array}\right) .
$$

The general solution

$$
W(t, \Theta)=U(t) \cdot \Theta \cdot V(t), \quad \Theta \in \mathbb{R}^{2 \times 2}
$$

of the matrix Cauchy problem

$$
Z^{\prime}(t)=A Z(t)+Z(t) B, \quad Z(0)=\Theta
$$

is determined $U(t)$ and $V(t)$ - normal $\left(U(0)=I_{2}, V(0)=I_{3}\right)$ fundamental matrices:

$$
U(t)=\left(\begin{array}{cc}
\cos t+\sin t & -2 \sin t \\
\sin t & \cos t-\sin t
\end{array}\right)
$$


and

$$
V(t)=\left(\begin{array}{cc}
\cos 2 t-\sin 2 t & -\sin 2 t \\
2 \sin 2 t & \cos 2 t+\sin 2 t
\end{array}\right)
$$

Denote the

$$
\Xi^{(1)}=\left(\begin{array}{ll}
1 & 0 \\
0 & 0
\end{array}\right), \quad \Xi^{(2)}=\left(\begin{array}{cc}
0 & 0 \\
1 & 0
\end{array}\right), \ldots, \Xi^{(4)}=\left(\begin{array}{cc}
0 & 0 \\
0 & 1
\end{array}\right)
$$

be a standard basis of the space $\mathbb{R}^{2 \times 2}$. The matrix $\mathcal{Q}=0$ defines a pseudoinverse matrix $\mathbb{Q}^{+}=0$ and the orthogonal projections $P_{Q_{Q}}=P_{Q^{*}}=I_{4}$. Since $P_{Q^{*}} \neq 0$, so far in the problem of constructing $2 \pi$-periodic solutions of matrix equations of Duffing type (3.1) holds critical case, and the condition (2.3) holds, hence the periodic problem for the matrix equations of Duffing type (3.1) has a solution of the form

$$
Z_{0}\left(t, \Theta_{r}\right)=W\left(t, \Theta_{r}\right)+G[F(s) ; \mathcal{A}](t), \quad \Theta_{r}=\left(\begin{array}{cc}
c_{1} & c_{3} \\
c_{2} & c_{4}
\end{array}\right),
$$

where

$$
G[F(s) ; \mathcal{A}](t)=K[F(s)](t) .
$$

The equation (2.7) for generating constants for the periodic problem for the matrix equations of Duffing type (3.1) has nontrivial real solution

$$
c_{r}^{*}=\left(-\frac{5}{16}-\frac{9}{32} \quad \frac{3}{32}-\frac{3}{32}\right)^{*} .
$$

Thus, we obtained matrix

$$
B_{0}=\pi\left(\begin{array}{rrrr}
-\frac{123}{256} & -\frac{49}{2048} & -\frac{481}{2048} & \frac{433}{1024} \\
\frac{49}{4096} & -\frac{1033}{2048} & -\frac{433}{2048} & \frac{385}{2048} \\
\frac{481}{4096} & -\frac{433}{2048} & -\frac{1465}{2048} & \frac{817}{2048} \\
\frac{433}{4096} & -\frac{385}{4096} & -\frac{817}{4096} & -\frac{81}{256}
\end{array}\right)
$$

and the orthogonal projections $P_{Q_{Q}}=P_{Q^{*}}=0$ enable one to verify the condition $P_{\mathscr{B}_{0}^{*}} P_{Q_{d}^{*}}=0$, which guarantee the unique solvability of the stated the periodic problem for the matrix equations of Duffing type (3.1), that for $\varepsilon=0$, turns into the the generating solution

$$
Z_{0}\left(t, \Theta_{r}^{*}\right)=W\left(t, \Theta_{r}^{*}\right)+G[F(s) ; \mathcal{A}](t), \quad \Theta_{r}^{*}=\left(\begin{array}{cc}
-\frac{5}{16} & \frac{3}{32} \\
-\frac{9}{32} & -\frac{3}{32}
\end{array}\right) .
$$

The matrix $\mathscr{B}_{0}$, which plays the key role in the critical case, is obtained in explicit form and coincides with the derivative of the equations for generating constants (2.7) for matrix boundary value problem (1.1), (1.2). We differentiate solvability condition (2.8):

$$
\frac{\partial}{\partial c_{r}} P_{\mathcal{Q}_{d}^{*}} \mathcal{M}\left\{\mathscr{L} K\left[\Phi\left(Z_{0}\left(s, \Theta_{r}^{*}\right), s, 0\right)\right](\cdot)\right\}
$$




$$
\begin{aligned}
& =\left.\frac{\partial}{\partial c_{r}} P_{Q_{d}^{*} \mathcal{M}}\{\mathscr{L} K[\Phi(Z(s, \varepsilon), s, \varepsilon)](\cdot)\}\right|_{\varepsilon=0} \\
& =\frac{\partial}{\partial c_{r}} P_{Q_{d}^{*} \mathcal{M}}\left\{\mathscr { L } K \left[\Phi\left(Z_{0}\left(s, \Theta_{r}^{*}\right), s, 0\right)+D\left[Z_{0}\left(s, \Theta_{r}^{*}\right) ; X(s, \varepsilon)\right]\right.\right. \\
& +R(Z(s, \varepsilon), s, \varepsilon)](\cdot)\}\left.\right|_{\varepsilon=0} \\
& =\left.\frac{\partial}{\partial c_{r}} P_{\mathcal{Q}_{d}^{*} \mathcal{M}}\left\{\mathscr{L} K\left[D\left[Z_{0}\left(s, \Theta_{r}^{*}\right) ; W\left(s, \Theta_{r}(\varepsilon)\right)\right]\right](\cdot)\right\}\right|_{\varepsilon=0} \\
& =\frac{\partial}{\partial c_{r}} P_{\mathcal{Q}_{d}^{*} \mathcal{M}} \mathcal{M}\left\{\mathscr { L } K \left[D\left[Z_{0}\left(s, \Theta_{r}^{*}\right) ; U(s) \Theta_{r}(\varepsilon) V(s)\right]=\left[\begin{array}{llll}
\mathcal{B}_{0}^{(1)} & \mathcal{B}_{0}^{(2)} & \ldots & \mathcal{B}_{0}^{(r)}
\end{array}\right] .\right.\right.
\end{aligned}
$$

The last equation is the matrix

$$
\mathcal{B}_{0}=\frac{\partial}{\partial c_{r}} P_{Q_{d}^{*} \mathcal{M}}\left\{\mathscr{L} K\left[\Phi\left(Z_{0}\left(s, \Theta_{r}^{*}\right), s, 0\right)\right](\cdot)\right\}
$$

which implies (by analogy with boundary value problem (1.1), (1.2) of the first order) that the condition $P_{\mathcal{B}_{0}^{*}} P_{Q_{d}^{*}}=0$ is equivalent to the fact that the $\operatorname{root} \Theta_{r}^{*} \in \mathbb{R}^{\alpha \times \beta}$ of equation for generating constants (2.7) is simple.

The method for construction of solvability conditions and construction of the generalized Green operator for Noetherian linear boundary value problem for the matrix differential equations and solvability conditions and the scheme for constructing solutions of nonlinear Noetherian boundary value problem for matrix differential equation can be generalized to boundary value problem for the matrix differential equations in various critical and noncritical cases $[6,16]$, in particular, to autonomous boundary value problems $[2,12]$ and nonlinear Noetherian second-order boundaryvalue problems in the critical case [11]. The method for construction of solvability conditions and construction of the generalized Green operator for Noetherian linear boundary value problem for the matrix differential equations and solvability conditions and the scheme for constructing solutions of nonlinear Noetherian boundary value problem for matrix differential equation can be generalized to boundary value problem for the matrix differential-algebraic equations [10].

\section{ACKNOWLEDGEMENT}

This work was financially supported by the State Foundation for Basic Research. Number of state Registered 0109U000381.

\section{REFERENCES}

[1] R. Bellman, Introduction to Matrix Analysis. Society for Industrial and Applied Mathematics. SIAM, 1970.

[2] A. Boichuk and S. Chuiko, "Autonomous weakly nonlinear boundary-value problems," Differents. Uravn, vol. 28, pp. 1668-1674, 1992. 
[3] A. Boichuk and S. Krivosheya, "Criterion of the solvability of matrix equations of the Lyapunov type," Ukrainian Mathematical Journal, vol. 50, no. 8, pp. 1162-1169, 1998, doi: 10.1007/BF02513089.

[4] A. Boichuk and S. Krivosheya, "A critical periodic boundary value problem for a matrix Riccati equation,” Differential Equations, vol. 37, no. 4, pp. 464-471, 2001, doi: 10.1023/A:1019267220924.

[5] A. Boichuk and A. Samoilenko, Generalized inverse operators and Fredholm boundary-value problems. Walter de Gruyter, 2004.

[6] A. Bondarev and V. Laptinskii, "Multipoint boundary value problem for the Lyapunov equation in the case of strong degeneration of the boundary conditions," Differential Equations, vol. 47, no. 6, pp. 778-786, 2011, doi: 10.1134/S0012266111060036.

[7] A. Chuiko, "Domain of convergence of an iteration procedure for a weakly nonlinear boundaryvalue problem," Nonlinear Oscillations, vol. 8, no. 2, pp. 277-287, 2005, doi: 10.1007/s11072005-0056-0.

[8] S. Chuiko, "Domain of convergence of an iterative procedure for an autonomous boundary-value problem," Nonlinear Oscillations, vol. 9, no. 3, pp. 405-422, 2006, doi: 10.1007/s11072-0060053-y.

[9] S. Chuiko, "On the solution of the matrix Sylvester equation," Visnyk Odesskogo Natsionalnogo Universitety (Math. and Mech.), no. vol. 19, Issue 1 (21), pp. 49-57, 2014.

[10] S. Chuiko, "The Green's operator of a generalized matrix differential-algebraic boundary value problem," Siberian Mathematical Journal, vol. 56, no. 4, pp. 942-951, 2015, doi: 10.17377/smzh.2015.56.417.

[11] S. Chuiko, A. A. Boichuk, and I. Boichuk, "Nonlinear Noetherian second-order boundary-value problems in the critical case," Journal of mathematical sciences, vol. 198, no. 3, pp. 351-360, 2014.

[12] S. Chuiko and I. Boichuk, "Autonomous Noetherian boundary-value problem in the critical case," Nonlinear Oscillations, vol. 12, no. 3, pp. 417-428, 2009, doi: 10.1007/s11072-010-0085-1.

[13] F. Gantmakher, Matrix theory. Nauka, 1988.

[14] E. Grebenikov and Y. Ryabov, Constructive Methods of Analysis of Nonlinear Systems. Nauka, 1979.

[15] L. Kantorovich and G. Akilov, Functional Analysis. Nauka, 1977.

[16] V. Laptinskii and D. Rogolev, "Constructive methods for obtaining the solution of the periodic boundary value problem for a system of matrix differential equations of Riccati type," Differential Equations, vol. 47, no. 10, pp. 1412-1420, 2011, doi: 10.1134/S0012266111100053.

[17] I. Malkin, Some Problems of the Theory of Nonlinear Oscillations. Gostekhizdat, 1956.

\section{Author's address}

\section{S. Chuiko}

Donbass State Pedagogical University, Department of Physics and Mathematics, Batyuk St., 84112 Slaviansk, Ukraine

E-mail address: chujko-slaveinbox.ru 